\title{
3-P-033 Poster Sessions
}

\section{Identification of a fluoxetine-target network in major depressive disorder}

\author{
Takashi Shiromizu $^{1}$, Koki Miura', Yuhei Nishimura ${ }^{1}$
}

${ }^{1}$ Dept. Integrative Pharm., Grad. Sch. Med., Mie Univ.

Background: While the efficacy of selective serotonin reuptake inhibitor (SSRI) has been widely accepted in major depressive disorder (MDD), mechanisms of the therapeutic action in the efficacy remain to be fully elucidated. The purpose of this study was to identify the SSRI-target network that might be relevant to the therapeutic mechanism.

Methods: From a public database, we downloaded transcriptome datasets (MDD patients and model mice) and analyzed the transcriptome response of fluoxetine (FLX). We analyzed these transcriptome datasets using weighted gene co-expression network analysis to identify networks significantly relevant to FLX-responsive MDD mice and patients. In order to ascertain whether the identified network worked also in vivo, we performed in situ hybridization using zebrafish larvae treated FLX.

Results: We were able to identify three gene co-expression networks significantly relevant to FLX-responsive MDD mice and patients. Comparison of the three networks revealed a common network consisting of 26 genes, including BDNF and RGS4. Both BDNF and RGS4 have been related to the therapeutic effects of SSRI.

Conclusions: The gene co-expression network identified in this study may be relevant to the therapeutic mechanism and the efficacy of SSRI. 